\title{
Restitution du permis de conduire. Examens biologiques à partir des cheveux
}

\author{
Hair analysis for driving licence
}

\section{Consensus de la Société Française de Toxicologie Analytique (SFTA)}

établi le 23 décembre 2004

\section{Bases légales}

Arrêté du 5 septembre 2001 fixant les modalités du dépistage des stupéfiants et des analyses et examens prévus par le décret $n^{\circ} 2001-751$.

Art. 10 - La recherche et le dosage des produits stupéfiants....s'effectuent en utilisant la technique dite «chromatographie en phase gazeuse couplée à la spectrométrie de masse»

Décret $n^{\circ} 2003-293$ du 31 mars 2003 relatif à la sécurité routière et modifiant le code de procédure pénale et le code de la route.

Art. 6 - Le préfet soumet à des analyses ou à des examens médicaux, cliniques et biologiques, notamment salivaires et capillaires

Loi $n^{\circ} 2003-495$ du 12 juin 2003 renforçant la lutte contre la violence routière.

Art. 1 - ....il résulte d'une analyse sanguine que le conducteur avait fait usage de substances ou de plantes classées comme stupéfiants......

Art. 35 - .....conduite en ayant fait usage de substances ou plantes classées comme stupéfiants.

\section{Base bibliographique}

1. Kintz P., Villain M., Cirimele V., Ludes B. Décret $n^{\circ}$ 2003-293 du 31 mars 2003. Restitution du permis de conduire à partir d'analyses de cheveux. Ann. Toxicol. Anal., 2003 ; 15(2) : 117-122.
2. Pépin G., Duffort G., Rommel N. et coll. Compilation de 3751 dosages sanguins de stupéfiants obtenus par 19 experts dans le cadre de la loi Gayssot. Ann. Toxicol. Anal. 2003 ; 15 (2) : 108-116.

3. Tagliaro F., De Battisti Z., Lubli G., Neri C., Manetto G., Marigo M. Integrated use of hair analysis to investigate the physical fitness to obtain the driving licence : a case-work study. Forensic Sci. Int. 1997 ; 84 : 129-135.

4. Sachs $\mathrm{H}$. Hair analysis as a basic for driving ability examination. Toxicorama $1994 ; 6,2: 11-17$.

5. Ricossa M.C., Bernini M., De Ferrari F. Hair analysis for driving licence in cocaine and heroin users. An epidemiological study. Forensic Sci. Int. $2000 ; 107$ : 301-308.

\section{Base théorique}

La mise en évidence de la conduite d'un véhicule sous l'influence de stupéfiants par des examens urinaires et sanguins est réalisée depuis septembre 2001. Les modalités du suivi médical d'un sujet ayant été déclaré positif sont maintenant envisagées. Rappelons que le décret $n^{\circ} 2003-293$ du 31 mars 2003 autorise pour cela le prélèvement de cheveux.

Selon la loi française, le sujet dont le permis de conduire a été suspendu pour conduite sous l'influence de stupéfiants ne peut en re-disposer qu'après passage devant une commission. Le rôle de cette commission est de vérifier que le sujet ne consomme plus de substances 
illicites depuis plusieurs mois. Elle utilise pour cela des tests cliniques et des examens toxicologiques.

Il est admis par la communauté scientifique que l'analyse des urines ne reflète, en général, qu'une exposition récente, datant de quelques jours. Au contraire, l'analyse d'une mèche de cheveux permet de mettre en évidence des expositions chroniques ou répétées, augmentant donc de façon importante la fenêtre de détection des stupéfiants. Les résultats donnent des renseignements sur le profil de consommation pendant plusieurs mois en fonction de la longueur des cheveux, son importance et son évolution : la consommation de stupéfiants ayant entraîné la suspension du permis de conduire a-t-elle cessé ou se poursuit-elle ?

Cette approche toxicologique de la restitution du permis de conduire est appliquée en routine en Allemagne et en Italie.

\section{Base analytique}

La méthode analytique retenue est la chromatographie en phase gazeuse couplée à la spectrométrie de masse (CPG/SM), à la fois sensible et spécifique, déjà référencée comme technique officielle pour la détection des stupéfiants dans le sang des conducteurs (Code de procédure pénale et Code de la route). La chromatographie en phase liquide couplée à la spectrométrie de masse en tandem (CL-SM/SM) est aussi envisageable.

\section{Prélèvement des cheveux}
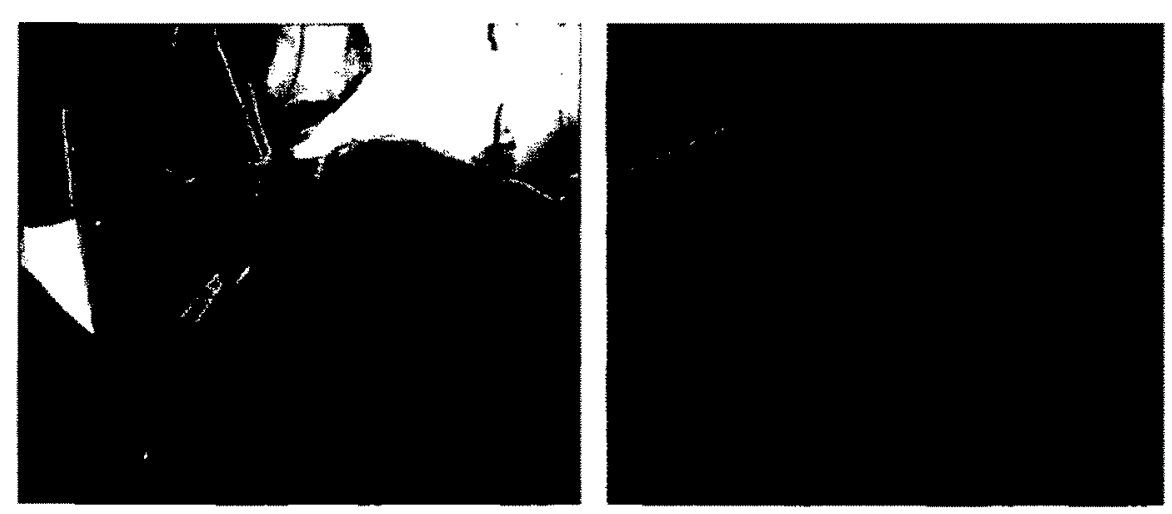

Quand : à n'importe quel moment, de préférence sur cheveux lavés

Combien : 4 mèches d'environ 40-50/ cheveux pour recherche des 4 familles de stupéfiants

Où : en vertex postérieur, c'est-à-dire sur l'arrière et le haut du crâne

\section{Comment :}

- orienter la mèche en maintenant les cheveux ensemble à l'aide d'une cordelette nouée à $1 \mathrm{~cm}$ de la racine ; l'orientation est nécessaire pour pouvoir segmenter les cheveux et ainsi retracer l'historique des faits.

- couper avec des ciseaux, de façon nette, le plus près possible de la peau.

\section{Ne pas arracher les cheveux! \\ Ne pas mettre de ruban adhésif!}

- conserver dans une enveloppe ou un tube sec, à température ambiante

\section{Ne pas réfrigérer, ne pas congeler!}

Il sera demandé à l'intéressé si un traitement cosmétique a été réalisé depuis la date des faits.

La procédure analytique, entièrement validée, devra comporter une étape de décontamination.

En fonction de la longueur des cheveux, du délai écoulé entre les faits délictueux et la dernière coupe de cheveux, 2 segments seront analysés d'emblée.

Les composés à rechercher et à doser, ainsi que leur seuil de positivité, sont proposés dans le tableau ci-dessous.

\section{Coût}

Recherche et dosage d'une famille :

$120 € \mathrm{HT}(143,52 € \mathrm{TTC})$

Recherche et dosage de 2 familles:

$240 € \mathrm{HT}(287,04 € \mathrm{TTC})$

Recherche et dosage de 3 ou 4 familles :

$300 € \mathrm{HT}(358,80 € \mathrm{TTC})$

\begin{tabular}{|c|c|c|}
\hline Famille & Molécule & Seuil de positivité \\
\hline Opiacés & $\begin{array}{c}\text { morphine, codéine, 6-acétylmorphine } \\
\text { codéthyline, pholcodine }\end{array}$ & $0,5 \mathrm{ng} / \mathrm{mg}$ \\
\hline Cocaïne & cocaïne, benzoylecgonine, cocaéthylène & $0,5 \mathrm{ng} / \mathrm{mg}$ \\
\hline Amphétamines & $\begin{array}{c}\text { amphétamine, méthamphétamine } \\
\text { MDA, MDMA, MDEA }\end{array}$ & $0,5 \mathrm{ng} / \mathrm{mg}$ \\
\hline Cannabis & THC, CBD & $0,1 \mathrm{ng} / \mathrm{mg}$ \\
\hline
\end{tabular}

La prise d'essai pour ces seuils est fixée à $30 \mathrm{mg}$.

Pour le cannabis, toute demande de confirmation devra comporter un dosage spécifique de THC-COOH (métabolite spécifique, marqueur d'une consommation de cannabis) par GC-MS/MS 